\title{
Segmented Regression via the Shape Language Modeling for Multi-Slope Path-Loss Modeling
}

\author{
Dayan Adionel Guimarães
}

\begin{abstract}
The multi-slope law for the area-mean received signal power decay has been considered a more adequate choice for modeling and analyzing the propagation path-loss in wireless networks. This paper presents a method for fitting localmean signal power measurements to a non-increasing, two- or three-slope piece-wise linear function of the transmitter-receiver distance. The optimal fit is achieved by means of the shape language modeling (SLM) tool when configured to the first order spline fitting option, with two and three segments. Some results are presented and discussed, and guidelines are given in what concerns the strategy for sampling the received power levels.
\end{abstract}

Keywords-Multi-slope path-loss, segmented regression, shape language modeling.

\section{INTRODUCTION}

Planing and deploying wireless communication systems often demand accurate knowledge of the environment-dependent signal propagation characteristics, mainly in terms of largescale path-loss. Path-loss knowledge is crucial for determining placement and coverage of base stations, and for optimizing the network. To this end, there are numerous propagation models available [1]-[5], some of them adopted by software packages known as wireless network planning tools [6] for coverage (or propagation) prediction. Many propagation prediction models are derived from, or are based on the well-known log-distance path-loss model [7], for instance the Stanford University Interim (SUI) [8], [9]. The log-distance model states that the area-mean received power decays linearly with the logarithm of the transmitter-receiver distance, with a slope determined by the environment morphology and topography. Other equally simple models dictate that the distancedependent area-mean received signal power decays following more than one slope. This is the case of the classic plane-earth two-ray model [10], in which the received signal power decay obeys a two-slope law whose breakpoint (where the slopes change inclination) lies at the location where the first Fresnel zone touches the ground [11].

Recently, the two-slope law and its multi-slope generalization have been put into focus, for instance in [12], where it has been demonstrated that the capacity and throughput analysis of a wireless network can be severely impacted if such a model is adopted, resulting in conclusions that significantly depart from those in which the conventional single-slope law is considered.

Dayan Adionel Guimarães is with the National Institute of Telecommunications (Inatel), PO Box 05, 37540-000 Santa Rita do Sapucaí - MG - Brazil (Tel:+55 (35) 3471 9227, Fax:+55 (35) 3471 9314, e-mail: dayan@inatel.br)

This work was partially supported by RNP, with resources from MCTIC Grant No. No 01245.010604/2020-14, under the 6G Mobile Communications Systems project of the Radiocommunication Reference Center (Centro de Referência em Radiocomunicações, CRR) of Inatel.
This is specially true in dense networks, e.g. in the fifthgeneration $(5 \mathrm{G})$ cellular systems, and Internet of things (IoT) and sensor networks with close apart nodes. The particular case of the dual-slope model has been shown to adhere to measurements in indoor [13] and outdoor [14]-[16] environments. In [17], a multi-slope fit from measured data has been proved to be suitable for path-loss modeling of the on-body channel, in the context of wireless body networks. The authors of [18] present experimental propagation measurements over the sea at $5.8 \mathrm{GHz}$, concluding that a multi-slope model is more appropriate than traditional models that use only one slope for non-line-of-sight transmissions. The signal power decay is modeled in [19] by a dual-breakpoint (three slopes) model as a function of the distance between a transmitter and an on-body sensor, and as a function of the body orientation angle for ultrawideband off-body communication channels in the frequency range between 3.5 and $6.5 \mathrm{GHz}$. In [20], the connection probability in vehicle-to-vehicle communications in urban scenarios is analyzed based on a dual-slope pathloss model. A ray-tracing based dual-slope model is proposed in [21] and claimed to be appropriate for evaluating the performance of millimeter-wave (mmWave) systems in dense urban environments. In [22], the influence of a dualslope path-loss model on the problem of user association in heterogeneous networks is investigated. A study about the capacity of cooperative vehicular ad hoc networks under a measurement-based dual-slope path-loss model is reported in [23]. Area-mean path-loss breakpoints were also observed in several test scenarios in a campaign based on mobile-tomobile channel measurements at $1.85 \mathrm{GHz}$ in dense-scattering suburban environments [24]. A recently-proposed model for the mmWave range also considers a two-slope path loss [25].

\section{A. Problem statement and typical solutions}

In the multi-slope path-loss model, the loss exponent associated to each slope and the breakpoints are derived from measured received powers, based on the log-distance model. However, this is not a trivial task because of the problem constraints: i) the area-mean received power must be a continuous function of the distance, by the nature of the distancedependent path-loss model. In other words, it is not expected that a propagation mechanism would produce discontinuities in the function that describes the variation of the area-mean received power with distance; ii) if the breakpoints of the multiple slopes were known, which is not reasonable to assume in practice, the application of the ordinary least squares (OLS) linear regression on the data set associated to each segment 
would result in a better fitting for each slope alone, but would produce discontinuities at the breakpoints and an overall useless regression result; iii) in a multi-slope model, it is common to consider that the first breakpoint appears at the distance from the transmitter in which the first Fresnel zone touches the ground. However, the actual first breakpoint distance may depart from the theoretical, since the presence of obstacles can move the touching point of the Fresnel zone to a distance closer to the transmitter. More breakpoints might show up in nondeterministic positions, depending on the particularities of each area under analysis. As a consequence, breakpoints often cannot be determined theoretically.

Typical approaches for solving the problem of multi-slope path-loss regression can resort to optimization techniques, for instance the ones adopted in [26]-[28], or to specialized software tools. In [26], a general approach of convex regression with adaptive classification is considered, and [27] applies particle swarm optimization (PSO) to the case of a multislope path-loss model. In terms of software packages, the shape language modeling (SLM) [28] is a versatile curve fitting tool based on least squares splines, which can be configured with first-order spline and other simple constraints to yield a piece-wise linear regression to input data. The SLM is implemented in Matlab and makes use of the Matlab optimization toolbox. The Origin package [29], which is a data analysis and graphing software application, has two-slope and three-slope built-in linear piece-wise fitting options. Another software tool for segmented regression is the joinpoint regression program (Jointpoint) [30], which has been developed to test whether or not an apparent change in a trend is statistically significant. The Joinpoint can also be applied to the multislope regression problem, but, likewise the Origin tool, its integration with automated analysis is not possible, owed to the lack of programming facilities similar to the Matlab.

\section{B. Contribution and organization of the paper}

This paper presents a method for gathering received signal power measurements and fitting the local-mean powers to a multi-slope path-loss model, using the SLM tool. Especial attention is paid to the fitting (or regression) process, since it is the most critical from the model parameterization standpoint. Besides simulated measurements and fitting results, the paper also provides guidelines for helping engineers and researchers to perform actual power measurements. Section II presents the multi-slope model. Sampling methods and the multi-slope regression are addressed in Section III. Section IV is devoted to numerical results and discussions, and Section V concludes the paper.

\section{MUlTi-SLOPE PATH-LOSS MODEL}

The multi-slope model is grounded on the two-ray plane earth model [10], or simply two-ray model, which has been found in [11] to suitably represent the signal attenuation in real scenarios departing from the idealized assumptions of the model. In this model, transmitter and receiver are in line-ofsight condition and lay on a plane-earth surface such that a reflected path towards the receiver is formed. Two path-loss regions separated by a breakpoint can be distinguished: before the breakpoint, the received signal power varies severely due to destructive and constructive combination between the direct and the reflected rays, whereas after the breakpoint it decreases linearly with the log-distance [11]. The breakpoint is at the distance $d_{\mathrm{c}}$, from the transmitter, in which the first Fresnel zone touches the ground. This distance, often referred to as the critical distance [12], [15], is given by $d_{\mathrm{c}} \approx 4 h_{t} h_{r} / \lambda$, where $h_{t}$ is the transmitting antenna height, $h_{r}$ is the receiving antenna height, and $\lambda$ is the carrier wavelength, all in meters.

In urban areas, the breakpoint might happen closer to the transmitter due to the presence of obstacles that hit the first Fresnel zone in a distance that might be far below $d_{c}$ [15], [16]. In such environments, the first breakpoint may be at, or even before the distance [16, eq. (2)]

$$
d_{\mathrm{bp}}=\frac{4\left(h_{t}-h_{\mathrm{avg}}\right)\left(h_{r}-h_{\mathrm{avg}}\right)}{\lambda},
$$

where $h_{\text {avg }}<h_{r}$ is the average height of obstacles between the transmitter and the receiver. For suburban areas, this average height is typically in the range of 6 to $15 \mathrm{~m} \mathrm{[31]-}$ [34], and for dense urban areas it typically lies in the range of 30 to $40 \mathrm{~m}$ [32], [33], [35].

More than one breakpoint may show-up from measured data [13]-[16], which gives rise to the multi-slope model [12]. In the case of three slopes, which can be readily specialized to any number of slopes, the area-mean received signal power, in $\mathrm{dBm}$, at a distance $d$ from the transmitter, is given by

$P(d)=\left\{\begin{array}{l}P\left(d_{0}\right)-10 n_{1} \log \left(\frac{d}{d_{0}}\right), d \leq \mathrm{BP}_{1} \\ P\left(d_{0}\right)-10 n_{2} \log \left(\frac{d}{d_{0}}\right)+\Delta_{1}, \mathrm{BP}_{1}<d<\mathrm{BP}_{2} \\ P\left(d_{0}\right)-10 n_{3} \log \left(\frac{d}{d_{0}}\right)+\Delta_{1}+\Delta_{2}, d \geq \mathrm{BP}_{2}\end{array}\right.$

where $n_{1}, n_{2}$ and $n_{3}$ are dimensionless loss exponents, $\mathrm{BP}_{1}$ and $\mathrm{BP}_{2}$ are the breakpoints, in meters, and $P\left(d_{0}\right)$ is the areamean received power, in $\mathrm{dBm}$, at a reference unobstructed distance $d_{0}$ from the transmitter, in meters, $\lambda \ll d_{0}<d_{\text {c }}$. For example, using the simplified form of the Friis equation, $P\left(d_{0}\right)=10 \log \left[P_{t} G_{t} G_{r}\left(\lambda / 4 \pi d_{0}\right)^{2}\right] \mathrm{dBm}$, where $P_{t}$ is the transmit power delivered to the antenna, in milli-watts $(\mathrm{mW})$, and $G_{t}$ and $G_{r}$ are the transmit and receive antenna dimensionless gains, respectively. Notice that this choice of $d_{0}$ contrasts with the value of $d_{0}>d_{\mathrm{c}}$ that is typically assumed in the conventional single-slope regression, a case in which $d_{0}$ must be placed after the first breakpoint that is typically considered to be at the critical distance. In such a case, the value of $P\left(d_{0}\right)$ is often determined from a single measurement of the areamean received power at a reference distance $d_{0}>d_{\mathrm{c}}$.

The constants $\Delta_{1}$ and $\Delta_{2}$ in (2) guarantee that successive slopes join at the breakpoints, and are given by

$$
\begin{aligned}
& \Delta_{1}=10\left(n_{2}-n_{1}\right) \log \left(\frac{\mathrm{BP}_{1}}{d_{0}}\right), \\
& \Delta_{2}=10\left(n_{3}-n_{2}\right) \log \left(\frac{\mathrm{BP}_{2}}{d_{0}}\right) .
\end{aligned}
$$

\section{SAMPLING AND SEGMENTED REGRESSION}

The characterization of received signal power variations can be made via samples collected during measurement cam- 
paigns. Since the number of samples $n$ needed to characterize the small-scale multipath fading is much larger than the number $N$ necessary for the analysis of large-scale propagation, here it is assumed that only the $N$ local-mean power measurements are retained, meaning that they describe both the distance-dependent signal attenuation and the power variation caused by obstacles, referred to as shadowing. Multipath fading is removed, which in practice is achieved by filtering instantaneous local measurements, or making measurements of average levels in each location of interest.

Two sampling methods are adopted here: method 1 considers equally-spaced samples in logarithmic-scaled distances, and method 2 adopts equally-spaced samples in linear-scaled distances. These methods can be interpreted with the help of Figure 1, where it is shown a hypothetical aerial view of a region of interest for the purpose of propagation analysis. The rectangles represent city blocks and the spaces between them are the streets. The transmitter is supposed to be in the middle block on the left, and instantaneous power measurements are supposed to be made along the path indicated by the solid line passing through some streets. It is assumed that the measurement platform is automated and has the following attributes: i) the measurement vehicle is equipped with a proper signal receiver, a time recorder, a positioning facility, for example a global positioning system (GPS) receiver, and a computer platform to store the measurements; ii) the spacial sampling spacing must be set to allow for capturing the short-term received signal power variations, meaning that the sampling frequency must be automatically varied according to the vehicle speed to save memory; iii) the storage is made so that measurements can be flexibly accessed. This latter attribute is of paramount importance. For example, suppose that method 2 is chosen, meaning that the area-mean powers in the scenario depicted by Figure 1 are retrieved from the storage in the locations indicated by the dark dots, which are at the intersections of the equidistant points from the transmitter (dashed arcs) and the measurement path (solid thick line). If the arcs are traced equidistantly in the log-distance, sampling at the intersections would correspond to method $\mathbf{1}$.

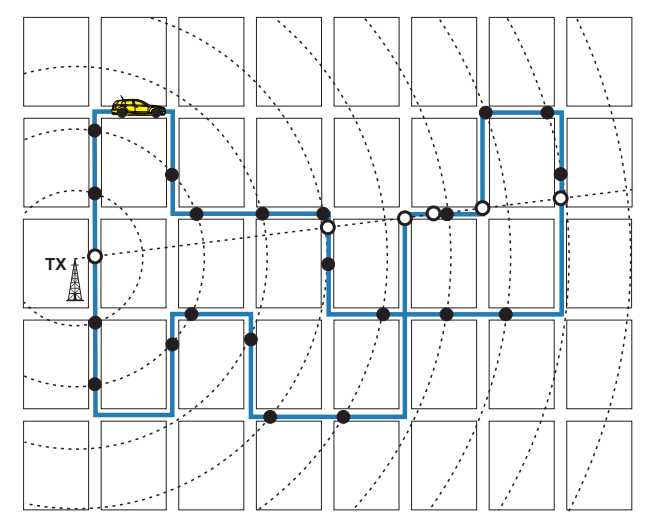

Fig. 1. Hypothetical partial aerial view of a measurement area, with equidistant points from the transmitter (dashed arcs), straight path departing from the transmitter (dashed straight line), measurement path (solid thick line). and local-mean power measurement points (dots).

Method 1 and method 2 have been created to help deter- mining if the scale of distances regarding the piece-wise linear fitting, which is logarithmic, should be used to determine the sampling spacing, or if this spacing should be governed by a linear scale. Method 1 produces samples with higher spatial density at the beginning of the linear-scaled distance range, decreasing this density as the distance approaches the end of the range. Method 2 produces sparse samples at the beginning of the logarithmic-scaled distances, increasing the sampling density as the distance approaches the end of the range.

Due to the attachment of the measurements to the time when they were gathered, it is possible to analyze the influence of time-varying whether or atmospheric conditions on the propagation characteristics of the environment. This is particularly useful in the mmWave range, in which the signal is strongly affected by rainfall. In this case, the measurement vehicle goes through the measurement path several times, at different moments of a day, or even at the same time of different days. In another situation, the local-mean received signal levels need to be analyzed along a straight line from the transmitter, in a given direction. In this case, the received powers must be read from the storage at points corresponding to the intersection of the straight line and the line associated to the measurement path, which is shown in Figure 1 by the non-filled dots. This would correspond to another sampling method in which the distance values are randomly distributed.

The segmented regression for multi-slope fitting has to be applied to spatially-correlated samples resulted from the shadowing process. However, the accuracy of the regression result is widened due to the spatial dependence among observations [36, p. 73]. This can be explained by the fact that, even for large sample sizes, the ratio between the actual number of samples $N$ and the effective sample size $N^{*}$ becomes $N / N^{*}=(1+\rho) /(1-\rho)$ [36, p. 73], being $\rho$ the correlation coefficient between neighbor samples. Then, strictly speaking, the reduction of the effective sample size due to an increased spatial correlation widens the confidence interval on the estimated parameter or statistic. To overcome this problem, $N$ is chosen such that neighbor samples have low correlation, using the rule-of-thumb

$$
N=\left\lceil\frac{d_{\max }-d_{0}}{d_{\mathrm{d}}}\right\rceil,
$$

where $d_{\max }$ is the upper limit of the transmitter-receiver distance, and $d_{\mathrm{d}}$ is the decorrelation distance that determines the degree of spatial correlation of the shadowing [14], both in meters. From this rule one can conclude that if more samples are needed for bettering the accuracy of the segmented regression in a given environment (fixed decorrelation distance), then a larger distance range must be considered.

It is also possible to improve the accuracy of the regression by taking more than one sample at each distance. For instance, if the value of $N$ computed from (5) is not sufficient for reaching the desired accuracy, and for some reason the measurement distance range cannot be increased, an effective number of samples $N^{\prime}$ can be obtained by taking $N^{\prime} / N$ samples, on average, for each of the $N$ distance points. The multiple samples per distance will be gathered at different locations, as illustrated by the dots on the arcs in Figure 1. In fact this 
is the most used method in practice, but care must be taken so that all samples belong to an area with certain homogeneity in terms of topography and morphology. For example, one should avoid mixing samples coming from a dense urban area with samples from a rural area, unless the propagation characterization is intended to infer about the joint influence of these two environments on the multi-slope path-loss modeling.

\section{RESULTS AND DISCUSSIONS}

This section presents segmented regression results using the SLM tool, whose engine function embedded in Matlab codes can be found in [37], applied to synthetic data generated by the simulation platform described in [38]. The parameters of the SLM engine function are described in [39].

Figures 2 and 3 give fitting results assuming that the localmean powers refer to a two-slope propagation scenario with loss exponents $n_{1}=2$ and $n_{2}=4$, shadowing standard deviation $\sigma=7 \mathrm{~dB}$, breakpoint $\mathrm{BP}=300 \mathrm{~m}$ and decorrelation distance $d_{\mathrm{d}}=10 \mathrm{~m}$. The reference distance is $d_{0}=1 \mathrm{~m}$, and the end of the distance range is $d_{\max }=1000 \mathrm{~m}$. The samples were processed with the SLM tool embedded in the two-lope fitting algorithm, and with the ordinary least squares linear regression (single-slope fitting). The number of samples is $N=500$, with 5 samples in each of the $N=100$ distances. Figure 2 considers equally-spaced log-distances, whereas Figure 3 considers equally-spaced linear-distances.

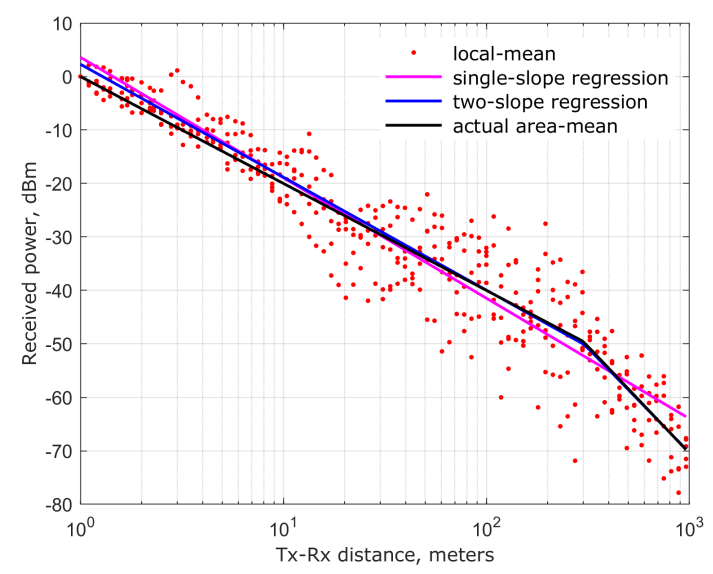

Fig. 2. Regressions to multiple measurement samples per log-spaced distances. Better viewed in color.

The parameters estimated from the scenario depicted in Figure 2 for the two-slope regression were $n_{1}=2.11$ and $n_{2}=3.91, \mathrm{BP}=304.99 \mathrm{~m}$, and $\sigma=5.70 \mathrm{~dB}$, with a coefficient of determination ${ }^{1} R^{2}=0.92$, and a mean residual sum of squares MRSS $=32.41$. The single-slope regression yielded $n=2.25, \sigma=5.94, R^{2}=0.92$ and MRSS $=35.21$.

Regarding the scenario considered in Figure 3, the estimated parameters were $n_{1}=2.24, n_{2}=4.19, \mathrm{BP}=322.35 \mathrm{~m}$ and

\footnotetext{
${ }^{1}$ The coefficient of determination $R^{2}$ is the proportion of the total variability in the dependent variable that is accounted for by the regression equation in the independent variables [40]. Using the $R^{2}$ alone may be not enough for assessing the quality of the regression, since fitted curves can have a high $R^{2}$ value, but wrong regression models [29], [40]. A solution is to combine the $R^{2}$ information with the mean residual sum of squares (MRSS).
}

$\sigma=6.85 \mathrm{~dB}$ for the two-slope regression, with $R^{2}=0.76$ and $\mathrm{MRSS}=46.86$. The single-slope regression yielded $n=2.91$, $\sigma=7.18 \mathrm{~dB}, R^{2}=0.73$ and MRSS $=51.33$.

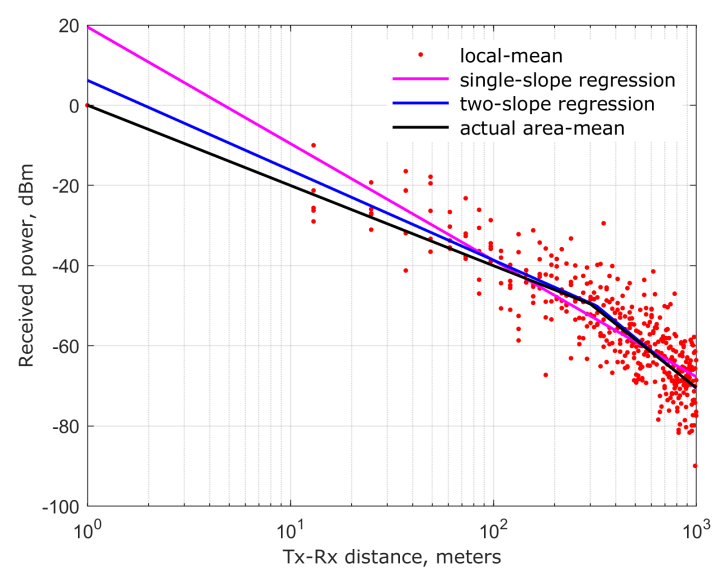

Fig. 3. Regressions to multiple measurement samples per linearly-spaced distances. Better viewed in color.

From these results it can be readily concluded that the conventional single-slope path-loss model is not capable of adequately describe the received signal power decay in the presence of a two-slope loss. The poor fits can be observed visually and by means of the $R^{2}$ and MRSS values.

It can be also noticed that samples equally spaced in logarithmic-scaled distances can produce better fits than samples equally spaced in linear scale. However, this is not a general conclusion, since the randomness of local-mean received powers, even in the same environment, turns the estimated parameters into random variables. In other words, if measurements are taken in the same region, but with the measuring vehicle traveling over different paths, different realizations of the local-mean powers would result, changing the values of the estimated multi-slope parameters. This fact leads to the need of associating other statistical metrics to the estimates, for example the confidence intervals on the loss exponents and breakpoint positions. One way of reducing variability consists of reducing the effect of measurement outliers, which can be done by means of robust and regularized fitting options of the SLM engine.

\section{Conclusions}

This paper presented a method for fitting local-mean signal power measurements to a non-increasing multi-slope piecewise linear function of the transmitter-receiver distance. The optimal fit was achieved by means of the shape language modeling tool when configured to the first order spline fitting option, with two and three segments. Some results were presented and discussed, and guidelines were given in what concerns the strategy for sampling the received power levels.

A future extended version of this paper could explore more test scenarios and path-loss parameters, as well as a deeper statistical analysis on the accuracy of the multi-slope pathloss modeling via segmented regression. This analysis can be 
made in light of confidence intervals on breakpoints and loss exponents, as well as of regression accuracy metrics.

Another subsequent research consists of capturing real received signal power data, perform multi-slope modeling and validate path-loss prediction under this model also based on real received signal levels across the area under analysis.

\section{REFERENCES}

[1] S. Hur, S. Baek, B. Kim, Y. Chang, A. F. Molisch, T. S. Rappaport, K. Haneda, and J. Park, "Proposal on millimeter-wave channel modeling for 5G cellular system," IEEE Journal of Selected Topics in Signal Processing, vol. 10, no. 3, pp. 454-469, April 2016.

[2] M. K. Samimi, T. S. Rappaport, and G. R. MacCartney, "Probabilistic omnidirectional path loss models for millimeter-wave outdoor communications," IEEE Wireless Communications Letters, vol. 4, no. 4, pp. 357-360, Aug 2015.

[3] C. Phillips, D. Sicker, and D. Grunwald, "A survey of wireless path loss prediction and coverage mapping methods," IEEE Communications Surveys Tutorials, vol. 15, no. 1, pp. 255-270, First 2013.

[4] T. K. Sarkar, M. C. Wicks, M. Salazar-Palma, and R. J. Bonneau, A Survey of Various Propagation Models for Mobile Communication Wiley-IEEE Press, 2003, pp. 239-307. [Online]. Available: http: //ieeexplore.ieee.org/xpl/articleDetails.jsp?arnumber $=5260004$

[5] M. F. Iskander, Z. Yun, and Z. Zhang, "Outdoor/indoor propagation modeling for wireless communications systems," in Antennas and Propagation Society International Symposium, 2001. IEEE, vol. 2, July 2001, pp. $150-153$ vol.2.

[6] The University of British Columbia, Department of Electrical and Computer Engineering , "Directory of Wireless System Planning Tools," Sep. 2016. [Online]. Available: http://rsl.ece.ubc.ca/planning.html

[7] T. Rappaport, Wireless Communications: Principles and Practice, 2nd ed. Upper Saddle River, NJ, USA: Prentice Hall PTR, 2002.

[8] P. Katev, "Propagation models for WiMAX at $3.5 \mathrm{GHz}$," in ELEKTRO, 2012, May 2012, pp. 61-65.

[9] A. Sulyman, A. Nassar, M. Samimi, G. MacCartney, T. Rappaport, and A. Alsanie, "Radio propagation path loss models for $5 \mathrm{G}$ cellular networks in the $28 \mathrm{GHz}$ and $38 \mathrm{GHz}$ millimeter-wave bands," Communications Magazine, IEEE, vol. 52, no. 9, pp. 78-86, September 2014.

[10] W. Lee, Mobile Communications Engineering. McGraw-Hill Professional Publishing, 1982.

[11] H. Xia, H. L. Bertoni, L. Maciel, A. Lindsay-Stewart, and R. Rowe, "Radio propagation characteristics for line-of-sight microcellular and personal communications," Antennas and Propagation, IEEE Transactions on, vol. 41, no. 10, pp. 1439-1447, Oct 1993.

[12] X. Zhang and J. Andrews, "Downlink cellular network analysis with multi-slope path loss models," Communications, IEEE Transactions on, vol. 63, no. 5, pp. 1881-1894, May 2015.

[13] C. B. Andrade and R. P. F. Hoefel, "On indoor coverage models for industrial facilities." in The 7th International Telecommunications Symposium (ITS 2010), Manaus, AM, Brazil, September 2010.

[14] T. Abbas, K. Sjoberg, J. Karedal, and F. Tufvesson, "A Measurement Based Shadow Fading Model for Vehicle-to-Vehicle Network Simulations," International Journal of Antennas and Propagation, vol. 2015, pp. 190607-, 2015. [Online]. Available: http://lup.lub.lu.se/record/7761043/file/7761061.pdf

[15] L. Cheng, B. Henty, D. Stancil, F. Bai, and P. Mudalige, "Mobile vehicleto-vehicle narrow-band channel measurement and characterization of the $5.9 \mathrm{GHz}$ dedicated short range communication (DSRC) frequency band,' Selected Areas in Communications, IEEE Journal on, vol. 25, no. 8, pp. 1501-1516, Oct 2007.

[16] H. Masui, T. Kobayashi, and M. Akaike, "Microwave path-loss modeling in urban line-of-sight environments," Selected Areas in Communications, IEEE Journal on, vol. 20, no. 6, pp. 1151-1155, Aug 2002.

[17] A. Alomainy and Y. Hao, "Radio channel models for UWB body-centric networks with compact planar antenna," in Antennas and Propagation Society International Symposium 2006, IEEE, July 2006, pp. 2173-2176.

[18] J. C. Reyes-Guerrero, G. Sisul, and L. A. Mariscal, "Measuring and estimating the propagation path loss and shadowing effects for marine wireless sensor networks at $5.8 \mathrm{GHz}$," in Telecommunications Forum (TELFOR), 2012 20th, Nov 2012, pp. 323-226.

[19] A. A. Goulianos, T. W. C. Brown, B. G. Evans, and S. Stavrou, "Wideband power modeling and time dispersion analysis for UWB indoor off-body communications," IEEE Transactions on Antennas and Propagation, vol. 57, no. 7, pp. 2162-2171, July 2009.
[20] R. Chen and Z. Zhong, "Analysis on v2v connectivity under dual-slope path loss model in urban scenarios," in General Assembly and Scientific Symposium (URSI GASS), 2014 XXXIth URSI, Aug 2014, pp. 1-4.

[21] Y. Chang, S. Baek, S. Hur, Y. Mok, and Y. Lee, "A novel dual-slope mmwave channel model based on 3d ray-tracing in urban environments," in Personal, Indoor, and Mobile Radio Communication (PIMRC), 2014 IEEE 25th Annual International Symposium on, Sept 2014, pp. 222-226.

[22] N. Garg, S. Singh, and J. Andrews, "Impact of dual slope path loss on user association in hetnets," in 2015 IEEE Globecom Workshops (GC Wkshps), Dec 2015, pp. 1-6.

[23] R. Chen, Z. Sheng, M. Ni, Z. Zhong, and D. G. Michelson, "Channel capacity under measurement-based model for cooperative vehicular ad hoc networks," in Antennas and Propagation USNC/URSI National Radio Science Meeting, 2015 IEEE International Symposium on, July 2015, pp. 302-303.

[24] Y. Ibdah and Y. Ding, "Mobile-to-mobile channel measurements at 1.85 GHz in suburban environments," IEEE Transactions on Communications, vol. 63, no. 2, pp. 466-475, Feb 2015.

[25] A. Ghosh, T. A. Thomas, M. C. Cudak, R. Ratasuk, P. Moorut, F. W. Vook, T. S. Rappaport, G. R. MacCartney, S. Sun, and S. Nie, "Millimeter-wave enhanced local area systems: A high-data-rate approach for future wireless networks," IEEE Journal on Selected Areas in Communications, vol. 32, no. 6, pp. 1152-1163, June 2014.

[26] L. A. Hannah and D. B. Dunson, "Multivariate Convex Regression with Adaptive Partitioning," Journal of Machine Learning Research, vol. 14, pp. 3261-3294, 2013. [Online]. Available: http://jmlr.org/papers/v14/ hannah13a.html

[27] Z. Raida et al., "Communication subsystems for emerging wireless technologies," Radioengineering, vol. 21, no. 4, pp. 1036-1049, Dec. 2012.

[28] J. D'Errico, "SLM: Shape language modeling," Apr. 2016. [Online]. Available: http://www.mathworks.com/matlabcentral/fileexchange/ 24443-slm-shape-language-modeling

[29] OriginLab Corporation, "Origin: A software application for data analysis and graphing," Nov. 2015. [Online]. Available: http://www. originlab.com/index.aspx?go=PRODUCTS/Origin

[30] Statistical Methodology and Applications Branch, Surveillance Research Program, National Cancer Institute, "Joinpoint Regression Program, Version 4.2.0," Apr. 2015. [Online]. Available: http://surveillance. cancer.gov/joinpoint/

[31] R. Arablouei and A. Ghorbani, "A new UTD-based model for multiple diffractions by buildings," in Microwave and Millimeter Wave Technology, 2002. Proceedings. ICMMT 2002. 2002 3rd International Conference on, Aug 2002, pp. 484-488.

[32] L. Piazzi and H. L. Bertoni, "A path loss formulation for wireless applications considering terrain effects for urban environments," in Vehicular Technology Conference, 1998. VTC 98. 48th IEEE, vol. 1, May 1998, pp. 159-163 vol.1.

[33] S. Saunders and F. Bonar, "Mobile radio propagation in built-up areas: a numerical model of slow fading," in Vehicular Technology Conference, 1991. Gateway to the Future Technology in Motion., 41st IEEE, May 1991, pp. 295-300.

[34] E. Lachat, J.-F. Wagen, and J. Li, "Effects of building heights on predictions in munich using a multiple vertical-knife-edges propagation model," in Vehicular Technology Conference, 1997, IEEE 47th, vol. 1, May 1997, pp. 261-265 vol.1.

[35] D. Crosby, S. Greaves, and A. Hopper, "The effect of building height variation on the multiple diffraction loss component of the walnschbertoni model," in Personal, Indoor and Mobile Radio Communications, 2003. PIMRC 2003. 14th IEEE Proceedings on, vol. 2, Sept 2003, pp. 1805-1809 vol.2.

[36] Y. Li, Modeling and Analysis of Spatially Correlated Data, ser. Frontiers of Statistics. Higher Education Press, 2009, ch. 4, pp. 72-98.

[37] D. A. Guimarães, "Simulation platform and codes for multi-slope segmented regression," May 2021. [Online]. Available: https://www. dropbox.com/s/tzndexzm919cvaf/Multi-slope_regression.zip?dl=0

[38] D. A. Guimarães and L. L. de Almeida Maia, "Plataforma para simulação de mecanismos de propagação em canais rádio móveis externos," Revista de Tecnologia da Informação e Comunicação, vol. 8, no. 2, pp. 51-68, 2018. [Online]. Available: http://rtic.com.br/index. $\mathrm{php} / \mathrm{rtic} /$ article/view/105

[39] J. D'Errico, "Shape Language Modeling (SLM) Tutorial," Apr. 2016. [Online]. Available: http://www.mathworks.com/matlabcentral/ fileexchange/24443-slm-shape-language-modeling/content/SLMtools/ $\mathrm{html} / \mathrm{slm}$ _tutorial.html

[40] G. Hahn, "The coefficient of determination exposed!" Chemical Technology, vol. 3, no. 10, pp. 609-614, Oct. 1973. 